\title{
DYNAMIC FAILURE IN DUCTILE POROUS MATERIALS
}

\author{
WANG ZE-PING \\ Institute of Applied Mechanics, Southwest Jiaotong University, Chengdu, Sichuan, 610031, P.R.C. \\ ZHENG JIAN \\ Laboratory of Non-linear Mechanics of Continuous Media, Institute of Mechanics, Chinese Academy \\ of Science, P.R.C.
}

\begin{abstract}
In this paper, a mathematical model of dynamic fracture in porous ductile materials under intense dynamic general loading is developed. The mathematical model includes the influence of inertial effects and material rate sensitivity, as well as the contribution of surface energy of a void and material work-hardening. In addition, the condition of the void compaction is considered as well. The threshold stresses for the void growth and compaction are obtained. A simple criterion for ductile fracture which is associated with material distention and plastic deformation is adopted. As an application of the theoretical model, the processes of two-dimensional spallation in LY12 aluminum alloy are successfully simulated by means of two-dimensional finite-difference Lagrangian code.
\end{abstract}

\section{INTRODUCTION}

DYNAMIC ductile fracture with different loading conditions such as high speed impact, explosive loading or dynamic tension of smooth or notched bar specimens is a consequence of the nucleation, growth and coalescence of voids in a triaxial stress field. In comparison to ductile damage under static loading, dynamic ductile damage is much more complex. The inertial effects, rate-dependence and thermal influence from rapid plastic deformation are the characteristics under intense dynamic loading. The literature on this subject is extensive. After investigating the behavior of dynamic damage and fracture in solids in detail, Curran and co-workers [1-4] established computational models called NAG (nucleation and growth) models for ductile and brittle fracture. In their models, two internal state variables $N$ (the number of microvoids or microcracks per unit volume) and $R$ (the average size of a microvoid or microcrack) are introduced to describe the processes of dynamic damage and fracture in solids. The NAG models have sufficient generality to include the statistical distribution of one or more variables such as porosity, void density, etc. but require numerous phenomenological constants that are difficult to obtain. Carroll and Holt [5] developed static and dynamic pore-collapse relations for ductile porous materials. The material was assumed to be rate insensitive and ideally plastic. They suggested that the effect of elastic compressibility in the matrix material is small, and can go immediately to the case of fully plastic deformation around the void. Johnson [6] applied Carroll and Holt's approach to void growth in the viscoplastic medium. Cochran and Banner [7] studied spallation in uranium using a simple theoretical model. Rajendran et al. [8] proposed a new dynamic failure model to describe void nucleation, growth and coalescence in ductile metals. Nash [9-10] directly used Rice's static model to model ductile fracture in triaxial states of stress. Cortes [11] adopted Carroll and Holt's assumption to investigate the growth of a microvoid under intense dynamic loading. Review articles [12-14] on dynamic ductile fracture explain in some detail the most relevant results of experimental and theoretical studies.

The inertial effects are the major feature of intense dynamic loading. Our theoretical analysis shows that its influence on the growth of ductile voids is significant. Since the problem is quite complicated when considering the inertial cffcets, most investigators neglected it.

The influence of deviatoric stresses is important for most dynamic ductile fractures. Although in the past several authors have studied the void growth problem under triaxiality conditions [15-18], they have limited their analysis to static loading, ignoring the influence of inertial effects. In this paper, we deal with damage and fracture in ductile materials under extremely high rates of general loading. A model of ductile dynamic fracture, in which the inertial effects, rate-dependence and the contribution of surface energy of voids are considered, is presented by 
means of the energy principles. The criterion for fracture is given by two quantities, distention $\alpha$ and the macroscopic effective plastic strain $E_{\text {eqv }}$.

To simplify theoretical analysis, we assume that the matrix material is incompressible during the void growth. We also assume that the void remains spherical at all times. These assumptions lead to a great simplification of the theoretical analysis, so that we obtain the exact relations for the void growth and collapse. As an application of the mathematical model presented in this work, the processes of two-dimensional spallation of LY12 aluminum alloy are simulated. Calculations are in fairly good agreement with experimental results.

\section{DESCRIPTION OF DYNAMIC DUCTILE FRACTURE}

The problem analysed in this paper is shown diagrammatically in Fig. 1. We assume that the porous material consists of a suspension of pores in a matrix of homogeneous isotropic solid ductile material which is subjected to an external stress $\Sigma_{i j}$, and that the porous material is statistically homogeneous and isotropic so that it can be effectively modeled by a homogeneous isotropic solid material. Meanwhile, we assume that the relation between pressure, specific volume, and specific internal energy for the matrix material is the same in the porous and nonporous conditions. Suppose that PVE relation (namely, equation of state) $P_{\mathrm{S}}\left(V_{\mathrm{S}}, e_{\mathrm{S}}\right)$, in the matrix material is given, the corresponding relation for the porous material is

$$
P=P_{\mathrm{S}}\left(V / \alpha, e_{\mathrm{S}}\right) / \alpha
$$

where $V$ is the macroscopic mean specific volume (averaged over the void and the matrix) and $e_{\mathrm{S}}$ is the specific internal energy in the solid surrounding the void. Variables with subscript " $S$ " are oncs in the matrix material. With these assumptions, we can study the void growth and compaction by considering a hollow sphere of the matrix material of inner radius $a$ and outer radius $b$; see Fig. 1(b). Distention $\alpha$ is defined as

$$
\alpha=\frac{b^{3}}{b^{3}-a^{3}}
$$

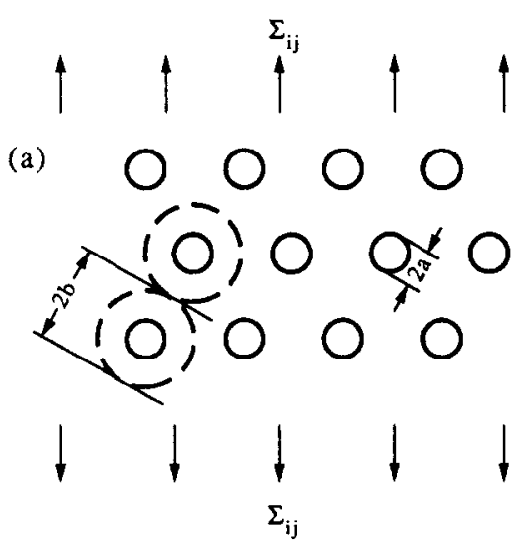

(b)

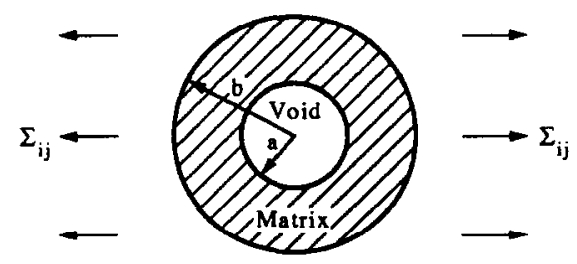

Fig. 1. (a) A body containing a homogeneous distribution of spherical voids of mean radius $a$ and mean spacing $b$ is subjected to an applied external stress $\Sigma_{\mathrm{ij}}$. (b) $A$ spherical element of material of radius $b$ containing a single void of radius $a$. 
We investigate the response of this hollow sphere to time-dependent external stress and zero internal pressure, and try to obtain the relations between the applied stress $\Sigma_{\mathrm{ij}}$ and distention $\alpha(t)$. We expect that these relations will adequately describe the void growth and compaction for the effective homogeneous material.

\subsection{Void growth and compaction rates}

Take the matrix material and void as a system; work done by the applied external stress $\Sigma_{\mathrm{ij}}$ is equal to the change of the system energy, namely,

$$
\Delta E_{\mathrm{K}}+\Delta E_{\mathrm{s}}+\Delta E_{\mathrm{i}}=W
$$

where $\Delta E_{\mathrm{K}}, \Delta E_{\mathrm{S}}, \Delta E_{\mathrm{i}}$ denote the changes of the kinetic energy, the surface energy of a void, and the internal energy in the system, respectively. $W$ denotes the work done by the applied external stress $\Sigma_{\mathrm{ij}}$.

As mentioned in the previous section, we neglect the initial elastic and elastic-plastic phase of the process, and begin to consider fully plastic deformation in the solid around the void.

Johnson's results [6] are used in this paper,

$$
\begin{gathered}
r^{3}=r_{0}^{3}-B(t) \\
B(t)=a_{0}^{3} \frac{\alpha_{0}-\alpha}{\alpha_{0}-1} \\
\frac{B(t)}{a^{3}}=\frac{\alpha_{0}-\alpha}{\alpha-1}, \quad \frac{B(t)}{b^{3}}=\frac{\alpha_{0}-\alpha}{\alpha} \\
\dot{r}=-\frac{\dot{B}(t)}{3 r^{2}},
\end{gathered}
$$

where "." denotes the differential with respect to time $t . r$ is the Eulerian radial position coordinate in the solid surrounding the void, $r_{0}$ is the initial value of the radius $r, \alpha_{0}$ is the initial value of the distention $\alpha$, and $a_{0}$ is the initial radius of the void.

Consider $\Delta E_{\mathrm{K}}, \Delta E_{\mathrm{S}}, \Delta E_{\mathrm{i}}$ and $W$, respectively. $\Delta E_{\mathrm{K}}$ is given by

$$
\Delta E_{\mathrm{K}}=E_{\mathrm{K}}(\alpha)-E_{\mathrm{K}}\left(\alpha_{0}\right) \text {, }
$$

where

$$
E_{\mathrm{K}}(\alpha)=\int_{a}^{b} \frac{1}{2} \dot{r}^{2} \rho 4 \pi r^{2} \mathrm{~d} r
$$

and $\rho$ is the density of the matrix material.

With the aim of eq. (2) and eqs (4)-(7), eq. (9) becomes

$$
E_{\mathrm{K}}(\alpha)=\left[\frac{4 \pi a_{0}^{3}}{9\left(\alpha_{0}-1\right)}\right] \frac{a_{0}^{2} \rho}{2\left(\alpha_{0}-1\right)}\left(\frac{\alpha_{0}-1}{\alpha-1}\right)^{1 / 3}\left[1-\left(\frac{\alpha-1}{\alpha}\right)^{1 / 3}\right] \dot{\alpha}^{2} .
$$

We also can obtain the expression for $\Delta E_{\mathrm{S}}$

where

$$
\Delta E_{\mathrm{S}}= \pm\left[E_{\mathrm{S}}(\alpha)-E_{\mathrm{S}}\left(\alpha_{0}\right)\right]
$$

$$
E_{\mathrm{S}}(\alpha)=\left[\frac{4 \pi a_{0}^{3}}{9\left(\alpha_{0}-1\right)}\right] \frac{9\left(\alpha_{0}-1\right)^{1 / 3} \gamma}{a_{0}}(\alpha-1)^{2 / 3} .
$$

$\gamma$ is the surface energy expended per unit area during the hole expanding. The upper sign + corresponds to void growth and the lower sign - corresponds to void compaction. Notice that in the following sections all the signs \pm correspond to void growth and compaction, respectively, unless they are explained.

The problem studied in the present paper is mainly concerned with the dynamic growth and compaction of a spherical void under intense dynamic loading. The dynamic growth of a ductile void can be approximately considered as an adiabatic process. In addition, we suppose that there 
is no heat source in the system. With these assumptions, the increment of the specific internal energy is given by

$$
\mathrm{d} e_{\mathrm{i}}=\frac{1}{\rho} \sigma_{\mathrm{jk}} \mathrm{d} \epsilon_{\mathrm{jk}}=\frac{1}{\rho}\left(\sigma_{\mathrm{jk}} \mathrm{d} \epsilon_{\mathrm{jk}}^{\mathrm{e}}+\sigma_{\mathrm{jk}} \mathrm{d} \epsilon_{\mathrm{jk}}^{\mathrm{p}}\right)
$$

where $\sigma_{\mathrm{jk}}$ is the stress tensor component, $\epsilon_{\mathrm{jk}}$ is the strain tensor component. Superscripts "e" and " $p$ " denote elastic and plastic parts of the strain tensor component. Here, the total strain increment is assumed to be the sum of elastic and plastic contributions:

$$
\mathrm{d} \epsilon_{\mathrm{jk}}=\mathrm{d} \epsilon_{\mathrm{jk}}^{\mathrm{e}}+\mathrm{d} \epsilon_{\mathrm{jk}}^{\mathrm{p}} .
$$

Based on the result that effects of elastic deformation in the solid around the void are much smaller than that of plastic deformation, the term $(1 / \rho) \sigma_{\mathrm{jk}} \mathrm{d} \epsilon_{\mathrm{jk}}^{\mathrm{e}}$ in eq. (13) can be ignored. Then eq. (13) can be written as

$$
\begin{aligned}
\mathrm{d} e_{\mathrm{i}} & =\frac{1}{\rho} \sigma_{\mathrm{jk}} \mathrm{d} \epsilon_{\mathrm{jk}}^{\mathrm{p}} \\
& =\frac{1}{\rho}\left(\sigma_{\mathrm{jk}}^{\prime}+\frac{1}{3} \sigma_{\mathrm{mm}} \delta_{\mathrm{jk}}\right) \mathrm{d} \epsilon_{\mathrm{jk}}^{\mathrm{p}} \\
& =\frac{1}{\rho}\left(\sigma_{\mathrm{jk}}^{\prime} \mathrm{d} \epsilon_{\mathrm{jk}}^{\mathrm{p}}+\frac{1}{3} \sigma_{\mathrm{mm}} \delta_{\mathrm{jk}} \mathrm{d} \epsilon_{\mathrm{jk}}^{\mathrm{p}}\right),
\end{aligned}
$$

where $\sigma_{\mathrm{ij}}^{\prime}$ is the deviatoric stress tensor component.

Since we assume that the matrix material is incompressible, $\mathrm{d} \epsilon_{\mathrm{kk}}^{\mathrm{p}}=0$. Therefore eq. (15) is reduced to

$$
\mathrm{d} e_{\mathrm{i}}=\frac{1}{\rho} \sigma_{\mathrm{jk}}^{\prime} \mathrm{d} \epsilon_{\mathrm{jk}}^{\mathrm{p}}=\frac{1}{\rho} \sigma_{\mathrm{eqv}} \mathrm{d} \epsilon_{\mathrm{eqv}}^{\mathrm{p}},
$$

where $\epsilon_{\mathrm{eqv}}$ and $\epsilon_{\mathrm{eqv}}^{\mathrm{p}}$ denote the effective stress and the effective plastic strain in the matrix material, respectively.

The matrix material is assumed to be rate sensitive, linear work-hardening, and viscoplastic. The constitutive relation is supposed to be

$$
\sigma_{\mathrm{eqv}}=Y_{0}+H \epsilon_{\mathrm{eqv}}^{\mathrm{p}}+\eta \dot{\epsilon}_{\mathrm{eqv}}^{\mathrm{p}},
$$

where $Y_{0}$ is the yield stress of the matrix material, $H$ is a linear work-hardening coefficient and $\eta$ is the material viscosity. Since we assume a plastic deformation process with spherical symmetry, the equivalent plastic strain $\epsilon_{\mathrm{eqv}}^{\mathrm{p}}$ is given by Johnson and Mellor [19]

$$
\epsilon_{\mathrm{eqv}}^{\mathrm{p}}= \pm 2 \ln \frac{r}{r_{0}} .
$$

The change of internal energy in the system is

$$
\Delta E_{\mathrm{i}}=\frac{1}{\rho} \int_{a}^{b}\left[\int_{0}^{\mathrm{C}_{\mathrm{eqv}}^{\mathrm{P}}} \sigma_{\mathrm{eqv}}\left(\epsilon^{\mathrm{P}}\right) \mathrm{d} \epsilon^{\mathrm{P}}\right] 4 \pi \rho r^{2} \mathrm{~d} r .
$$

By means of eqs (4)-(7) and eq. (17), we finally have

$$
\Delta E_{\mathrm{i}}=\left[\frac{4 \pi a_{0}^{3}}{9\left(\alpha_{0}-1\right)}\right]\left[F_{3}(\alpha)+F_{4}(\alpha)+F_{5}(\alpha) \dot{\alpha}\right]
$$

where

$$
\begin{gathered}
F_{3}(\alpha)= \pm 2 Y_{0}\left(\ln \frac{\alpha-1}{\alpha_{0}-1}+\alpha \ln \frac{\alpha}{\alpha-1}-\alpha_{0} \ln \frac{\alpha_{0}}{\alpha_{0}-1}\right) \\
F_{4}(\alpha)=\frac{4}{3} H\left[\left(\alpha-\alpha_{0}\right) F(\alpha)+\frac{\alpha_{0}}{2}\left(\ln \frac{\alpha_{0}}{\alpha}\right)^{2}-\frac{\alpha_{0}-1}{2}\left(\ln \frac{\alpha_{0}-1}{\alpha-1}\right)^{2}\right] \\
F(\alpha)=\int_{h_{0}}^{h_{1}} \frac{\ln (h+1)}{h} \mathrm{~d} h
\end{gathered}
$$




$$
\begin{gathered}
h_{0}=\frac{\alpha_{0}-\alpha}{\alpha-1}, \quad h_{1}=\frac{\alpha_{0}-\alpha}{\alpha} \\
F_{5}(\alpha)=\frac{2}{3} \eta\left(\ln \frac{\alpha-1}{\alpha}+\ln \frac{\alpha_{0}}{\alpha_{0}-1}\right) .
\end{gathered}
$$

Functions $F_{3}(\alpha), F_{4}(\alpha)$ and $F_{5}(\alpha)$ denote the influence of the yield stress of the matrix material, the linear work-hardening and the material viscosity on the increment of internal energy, respectively.

The increment of work per unit volume done by the applied external stress $\Sigma_{\mathrm{ij}}$ is given by

$$
\mathrm{d} w=\Sigma_{\mathrm{ij}} \mathrm{d} E_{\mathrm{ij}}
$$

where $E_{\mathrm{ij}}$ is the macroscopic deformation component which is defined in the same way as Gurson's [16] definition

$$
\dot{E}_{\mathrm{ij}}=\frac{1}{V} \int_{V} \dot{\epsilon}_{\mathrm{ij}} \mathrm{d} V .
$$

$V$ is the volume of the spherical element, $\dot{\epsilon}_{i j}$ is the microscopic rate of deformation field. With external boundary conditions put in terms of the macroscopic rate of deformation field $\dot{E}_{\mathrm{ij}}$,

$$
\left.v_{\mathrm{i}}\right|_{s}=\left.\dot{E}_{\mathrm{ij}} x_{\mathrm{j}}\right|_{s} \quad \text { (Cartesian coordinates), }
$$

where $v_{1}$ is the microscopic velocity field and $x_{1}$ is the position of a material point in Cartesian coordinates.

From eqs (26) and (27), we have

$$
\dot{E}_{\mathrm{ij}}^{\prime}=\dot{\epsilon}_{\mathrm{ij}}^{\prime}, \quad \dot{E}_{\mathrm{kk}}=\dot{V} / V
$$

where $\dot{E}_{\mathrm{ij}}^{\prime}$ and $\dot{c}_{\mathrm{ij}}^{\prime}$ are the deviatoric parts of the macroscopic and microscopic rates of deformation field, respectively.

The increment of work per unit volume also may be written

$$
\begin{aligned}
\mathrm{d} w & =\Sigma_{\mathrm{ij}} \mathrm{d} E_{\mathrm{ij}} \\
& =\left(\Sigma_{\mathrm{ij}}^{\prime}+\frac{1}{3} \Sigma_{\mathrm{kk}} \delta_{\mathrm{ij}}\right)\left(\mathrm{d} E_{\mathrm{ij}}^{\prime}+\frac{1}{3} \delta_{\mathrm{ij}} \mathrm{d} E_{\mathrm{nn}}\right) \\
& =\Sigma_{\mathrm{ij}}^{\prime} \mathrm{d} E_{\mathrm{ij}}^{\prime}+\frac{1}{3} \Sigma_{\mathrm{kk}} \mathrm{d} E_{\mathrm{nn}} \\
& =\Sigma_{\mathrm{eqv}} \mathrm{d} E_{\mathrm{eqv}}+\frac{1}{3} \Sigma_{\mathrm{kk}} \frac{\mathrm{d} V}{V},
\end{aligned}
$$

where $\Sigma_{\mathrm{ij}}^{\prime}$ is the macroscopic deviatoric stress, and $\Sigma_{\text {eqv }}, E_{\text {eqv }}$ denote the macroscopic effective stress and strain, respectively,

$$
\Sigma_{\text {eqv }}=\left(\frac{3}{2} \Sigma_{\mathrm{ij}}^{\prime} \Sigma_{\mathrm{ij}}^{\prime}\right)^{1 / 2}, \quad E_{\mathrm{eqv}}=\left(\frac{2}{3} E_{\mathrm{ij}}^{\prime} E_{\mathrm{ij}}^{\prime}\right)^{1 / 2}, \quad \frac{1}{3} \Sigma_{\mathrm{kk}}=-P .
$$

Using eqs (6) and (28), the following relations are given

$$
\mathrm{d} E_{\mathrm{eqv}}=\mathrm{d} \epsilon_{\mathrm{eqv}}^{\mathrm{P}}=\frac{2}{3} \frac{\mathrm{d} \alpha}{\alpha}, \quad \frac{\dot{V}}{V}=\frac{\dot{\alpha}}{\alpha} .
$$

With the help of eqs (29)-(31), work $W$ done by external stress is as follows

$$
\begin{aligned}
W & =\int_{\alpha_{0}}^{\alpha} \frac{4}{3} \pi b^{3} \mathrm{~d} w \\
& =\frac{4}{3} \pi \frac{a_{0}^{3}}{\alpha_{0}-1} \int_{\alpha_{0}}^{\alpha}\left(\frac{2}{3} \Sigma_{\text {eqv }}-P\right) \mathrm{d} \alpha .
\end{aligned}
$$

As the assumption of eq. (1), we also assume $\Sigma_{\text {eqv }}$ to be a function of distention $\alpha$, that is, $\Sigma_{\text {eqv }}=\Sigma_{\text {eqv }}(\alpha)$.

Substitution of eqs (8)-(12), eq. (20) and eq. (32) into eq. (3) gives

$$
F_{1}(\alpha) \dot{\alpha}^{2}+F_{5}(\alpha) \dot{\alpha}+F_{7}(\alpha)=0
$$


where

$$
\begin{gathered}
F_{1}(\alpha)=\frac{\rho a_{0}^{2}}{2\left(\alpha_{0}-1\right)}\left(\frac{\alpha_{0}-1}{\alpha-1}\right)^{1 / 3}\left[1-\left(\frac{\alpha-1}{\alpha}\right)^{1 / 3}\right] \\
F_{7}(\alpha)=F_{2}(\alpha)+F_{3}(\alpha)+F_{4}(\alpha)-3 \int_{\alpha_{0}}^{\alpha}\left(\frac{2}{3} \Sigma_{\text {eqv }}(\beta)-P(\beta)\right) \mathrm{d} \beta-F_{1}\left(\alpha_{0}\right) \dot{\alpha}_{0}^{2}-F_{2}\left(\alpha_{0}\right) \\
F_{2}(\alpha)= \pm \frac{9\left(\alpha_{0}-1\right)^{1 / 3} \gamma}{a_{0}}(\alpha-1)^{2 / 3} .
\end{gathered}
$$

Equation (33) is the relationship from which we obtain the rate-dependent response of the void growth and compaction under dynamic loading.

The terms in eq. (33) have a clear physical significance. The first term $F_{1}(\alpha) \dot{\alpha}^{2}-F_{1}\left(\alpha_{0}\right) \dot{\alpha}_{0}^{2}$ on the left of eq. (33) represents inertial resistance to the void growth and compaction. The second term $F_{5}(\alpha) \dot{\alpha}$ denotes the influence of the material viscosity. The third term $F_{7}(\alpha)$ is the sum of the total effects of the applied external stress, the change of the surface energy of voids, work-hardening and the yield stress in the solid surrounding the void on the void growth and compaction. These effects also can be isolated and studied in great depth.

From eq. (33), the void growth and compaction rates $\dot{\alpha}$ can be given

$$
\dot{\alpha}=\frac{1}{2 F_{1}(\alpha)}\left\{-F_{5}(\alpha) \pm \sqrt{\left[F_{5}(\alpha)\right]^{2}-4 F_{1}(\alpha) F_{7}(\alpha)}\right\},
$$

where $F_{1}(\alpha)>0, F_{5}(\alpha)>0$ (void growth), $F_{5}(\alpha)<0$ (void compaction). Since $\dot{\alpha}$ is a real variable, the following condition must be satisfied

$$
\left[F_{5}(\alpha)\right]^{2}-4 F_{1}(\alpha) F_{7}(\alpha) \geqslant 0 .
$$

If the inertial effects are neglected, from eq. (33), $\dot{\alpha}$ is reduced to

$$
\dot{\alpha}=-\left[F_{7}(\alpha)+F_{1}\left(\alpha_{0}\right) \dot{\alpha}_{0}^{2}\right] / F_{5}(\alpha) .
$$

Equation (38) is for both void growth and compaction. However, one must note that $F_{5}(\alpha)<0$ for the void compaction.

\subsection{Threshold stresses for dynamic growth and compaction of voids}

Define a quantity $\Sigma$ as

$$
\Sigma=\frac{2}{3} \Sigma_{\mathrm{eqv}}-P .
$$

Obviously, $\Sigma$ represents the total external stress acting on the spherical element.

We consider the condition of the void growth, namely, $\dot{\alpha} \geqslant 0$. Analysis of the condition of the void compaction is the same as that of the void growth. From eq. (37), the following inequality must be satisfied

$$
\Sigma(\alpha) \geqslant \frac{1}{3}\left[\frac{\mathrm{d} F_{2}(\alpha)}{\mathrm{d} \alpha}+\frac{\mathrm{d} F_{3}(\alpha)}{\mathrm{d} \alpha}+\frac{\mathrm{d} F_{4}(\alpha)}{\mathrm{d} \alpha}\right]
$$

Table 1. Material constants

\begin{tabular}{cccc}
\hline & Copper & Aluminum & PMMA* $^{*}$ \\
\hline$\rho_{0}\left(\mathrm{~g} / \mathrm{cm}^{2}\right)$ & 8.92 & 2.79 & 1.185 \\
$K_{0}(\mathrm{GPa})$ & 136.0 & 86.0 & 7.0 \\
$\Gamma$ & 1.94 & 1.7 & 1.0 \\
$\gamma_{0}(\mathrm{GPa})$ & 0.26 & 0.33 & \\
$\mu_{\mathrm{s}}(\mathrm{GPa})$ & 48.4 & 37.5 & \\
$\eta(\mathrm{GPa} \cdot \mu \mathrm{S})$ & 0.1 & 0.1 & \\
$\gamma\left(\mathrm{J} / \mathrm{m}^{2}\right)$ & 0.09 & 0.1 & \\
$\chi_{\text {crit }}$ & 1.45 & 1.4 & \\
$H(\mathrm{GPa})$ & 0.25 & 0.23 & \\
\hline
\end{tabular}

*PMM $\Lambda$ is treated in these calculations as a fluid (no shear strength). 


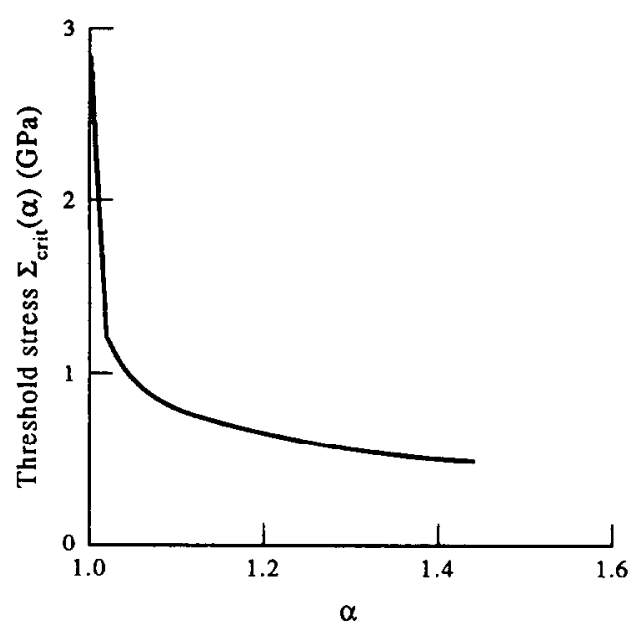

Fig. 2. The threshold stress $\Sigma_{\text {crit }}(\alpha)$ for the void growth decreases along with increase of distention $\alpha$.

With the help of eqs (21)-(22) and (36), inequality (40) becomes

$$
\Sigma(\alpha) \geqslant \frac{1}{3}\left[\frac{6 \gamma}{a_{0}}\left(\frac{\alpha_{0}-1}{\alpha-1}\right)^{1 / 3}+\frac{4 H}{3} F(\alpha)-\frac{8 H}{3} \frac{\alpha_{0}-1}{\alpha-1} \ln \frac{\alpha-1}{\alpha_{0}-1}+2 Y_{0} \ln \frac{\alpha}{\alpha-1}\right] .
$$
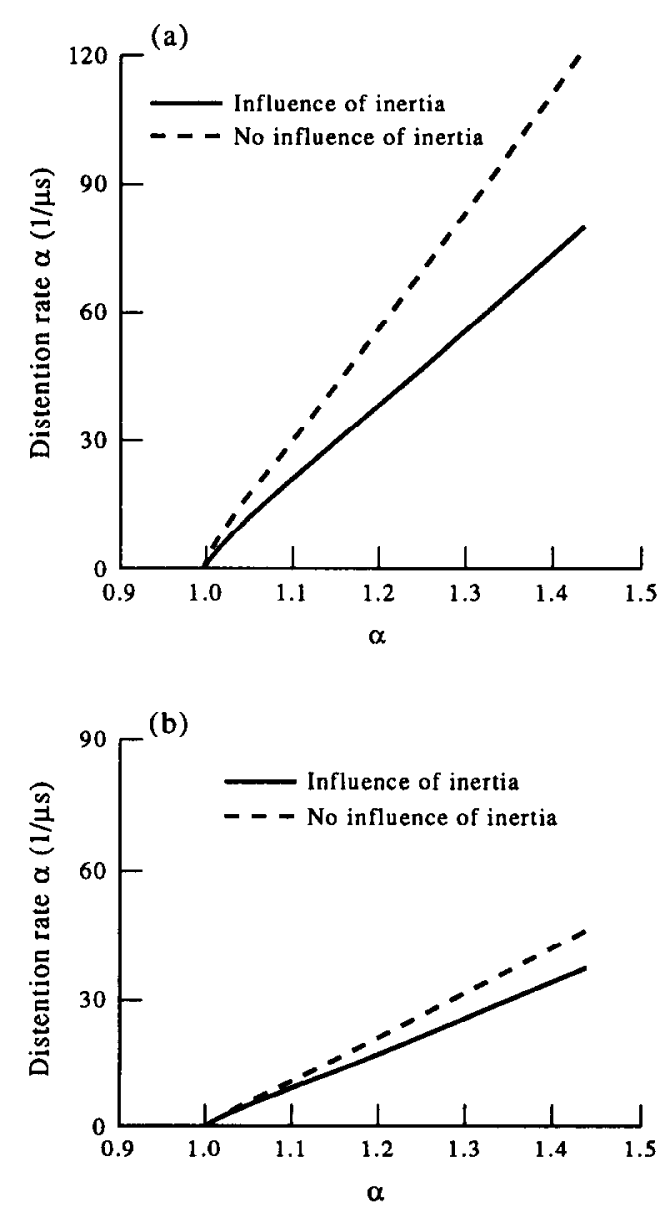

Fig. 3. Influence of inertia on the increment of distention rate $\dot{\alpha}$ under the different rates of loading. (a) $G=0.3$. (b) $G=0.1$. 
Let

$$
\Sigma_{\text {crit }}(\alpha)= \pm \frac{1}{3}\left[\frac{6 \gamma}{a_{0}}\left(\frac{\alpha_{0}-1}{\alpha-1}\right)^{1.3}+\frac{4 H}{3} F(\alpha)-\frac{8 H}{3} \frac{x_{0}-1}{\alpha-1} \ln \frac{\alpha-1}{\alpha_{0}-1}+2 Y_{0} \ln \frac{x}{\alpha-1}\right],
$$

where $\Sigma_{\text {crit }}(\alpha)$ are the threshold stresses for both dynamic growth and compaction of voids in general dynamic loading conditions. Sign + is for void growth and sign - is for void compaction.

The critical conditions that the applied external stress must satisfy for the void growth and compaction are that

$$
\begin{cases}\Sigma(\alpha)>\Sigma_{\text {crit }}(\alpha) & \text { (void growth) } \\ \Sigma(\alpha)<\Sigma_{\text {crit }}(\alpha) & \text { (void compaction) }\end{cases}
$$

\subsection{Numerical analysis}

In this section, pure copper is selected to be the material for the numerical analysis. The major material parameters used for calculations are listed in Table 1. In this section, only the situation of the void growth is considered. The approach for analysis of the void compaction is analogous.

The relation of the threshold stress $\Sigma_{\text {crit }}(\alpha)$ and distention $\alpha$ for the void growth in terms of eq. (42) is depicted in Fig. 2. It shows that the threshold stress $\Sigma_{\text {crit }}(\alpha)$ decreases quickly as distention $\alpha$ increases. This implies that the larger the ductile voids in the solid are, the smaller the stress needs for the void growth.

To investigate the effects of inertia, under the different rate loading, we numerically analyse the analytical eqs. (37) and (38) previously developed. In order to simplify the analysis, the material is assumed to be subjected to a linearly increasing external stress.

$$
\Sigma(\alpha)=\Sigma_{0}+G\left(\alpha-x_{0}\right),
$$

where $\Sigma_{0}=\Sigma_{\text {crit }}\left(\alpha_{0}\right)$ and $G$ are constants.

Figure 3 indicates that the influence of inertial effects becomes larger along with the increase of loading rates. $G=0.3,0.1$ equal approximately to the loading rates $10.0 \mathrm{GPa} / \mu \mathrm{s}$ and $1.0 \mathrm{GPa} / \mu \mathrm{s}$. respectively. The results of numerical analysis in Fig. 3 suggest that the influence of inertial effects plays an important role in the void growth under high rate loading conditions.

\subsection{Criteria for fracture}

Large numbers of experimental observations $[12,13,20]$ show that the mechanism of dynamic ductile fracture is not unique, and it varies with different microstructure of materials. Coalescence of voids is found, in general, to have two modes, that is, the direct impingement and that concerned with microcracks which are composed of much smaller microvoids. These two modes of coalescence of voids also were observed in our photomicrographs of recovered spalled specimens of pure copper and LY12 aluminum alloy (shown in Fig. 4). After investigating the ductile fracture of quenched and tempered steels, Hancock and Mackenzie [20] found that the large holes do not actually touch one another at failure initiation but are often joined by micro-cracks. Such cracks resulted from smaller-scale hole growth processes, probably based on carbides and small inclusions, and would seem to require a locally severe stress state and/or very high local strains. Shockey et al. [21] have measured the porosity developed in a notched tensilc bar for tensile tests on $\Lambda 553 \mathrm{~B}$ pressure vessel steel, and found that fracture occurs at the plastic strain of 1.0 and a final porosity of 0.03 . All the facts indicate that a single quantity porosity $\Phi$ (defined as $\Phi=(\alpha-1) / \alpha)$ is not sufficient to be the criterion for ductile fracture. The influence of plastic strain on fracture would be considered. Johnson [22] proposed a fracture surface,

Table 2. Experimental parameters

\begin{tabular}{cccccccc}
\hline & Impact velocity & \multicolumn{4}{c}{ Sample size $(\mathrm{mm})$} \\
Expt No. & $(\mathrm{m} / \mathrm{s})$ & Kind of gauge & $\mathrm{a}$ & $\mathrm{b}$ & $\mathrm{c}$ & $\mathrm{d}$ & $\mathrm{e}$ \\
\hline 1 & 356 & Carbon & 1.90 & 7.58 & 9.82 & 12.0 & 70.0 \\
2 & 362 & Manganin & 2.05 & 7.80 & 9.75 & 12.0 & 70.0 \\
3 & 207 & & 2.00 & 8.25 & 9.74 & 12.0 & 70.0 \\
\hline
\end{tabular}




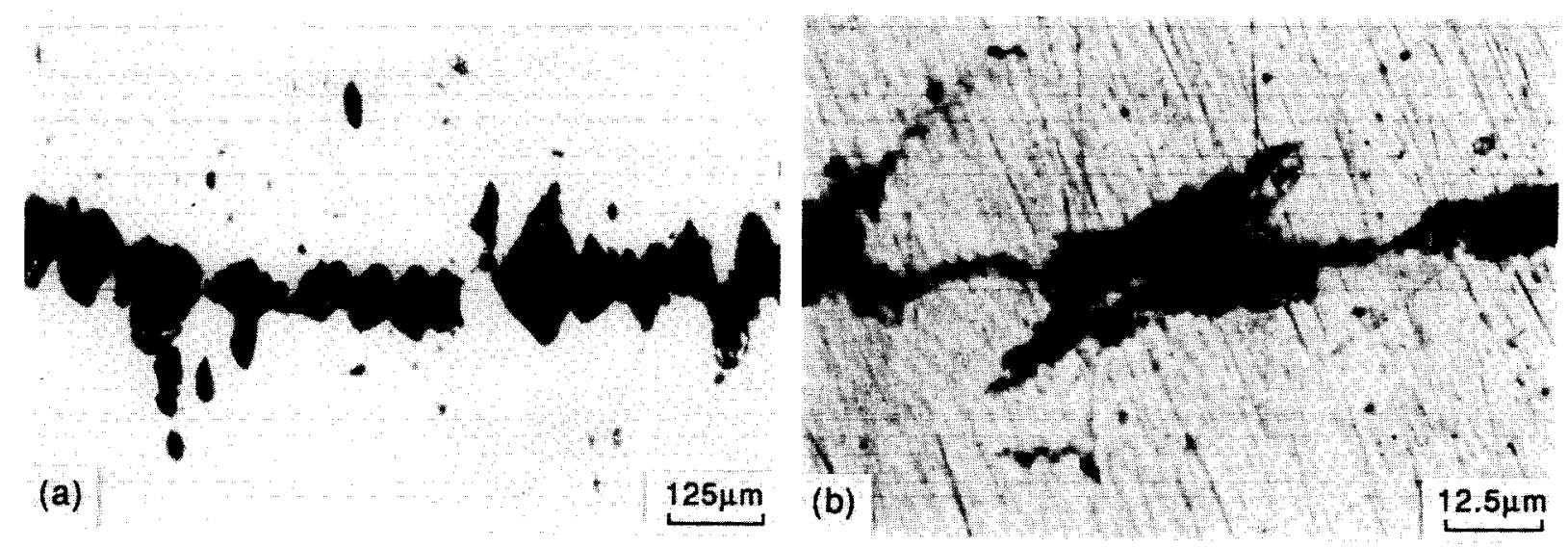

Fig. 4. Photomicrographs of recovered spalled specimens. (a) Direct impingement of large holes in pure copper specimen, (b) large holes concerned by microcracks in LY12 aluminum alloy sample. 
Impact surface

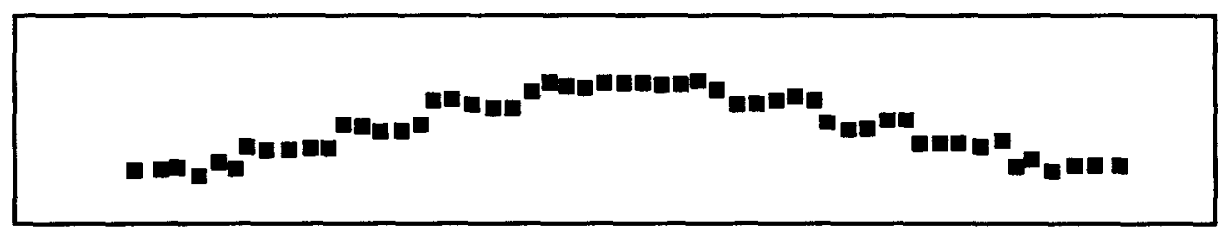

(a)

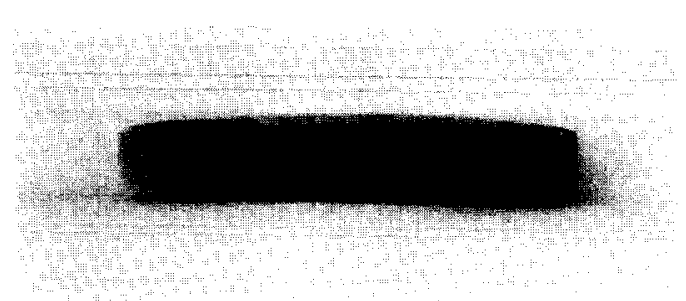

(b)

Fig. 8. Comparison of computed and observed spall surface in the specimen impacted by a tapered flyer (test 3). (a) Computed spall surface on cross section of LY12 aluminum alloy. (b) Cross-section of LY 12 aluminum recovered target. 


$$
\left(\begin{array}{c}
\Phi \\
\Phi_{\mathrm{f}}
\end{array}\right)^{2}+\left(\begin{array}{c}
\epsilon_{\mathrm{eqv}}^{\mathrm{p}} \\
\epsilon_{\mathrm{f}}
\end{array}\right)^{2}=1,
$$

as the criterion for fracture. Where $\Phi_{\mathrm{f}}$ and $\epsilon_{\mathrm{f}}$ are the critical porosity and effective plastic stain for fracture. The idealization of the fracture surface is employed in the present work. Define a fracture function:

$$
f\left(\alpha, E_{\text {eqv }}\right)=A .
$$

Equation (46) is the condition for dynamic ductile fracture in solids. Function $f\left(\alpha, E_{\text {eqv }}\right)$ may have different expression. $A$ is a constant which can be determined by experiments. Through eq. (46), ductile fracture is associated with distention $\alpha$ and plastic deformation $E_{\text {cqv }}$. This implies that not only the contribution of distention $\alpha$ but also local flow instability are taken into account for failure initiation. In the present study, a simple formation of $f\left(\alpha, E_{\text {eqv }}\right)$ is adopted.

$$
f\left(\alpha, E_{\text {cqv }}\right)=\frac{\alpha}{\alpha_{\text {crit }}}+\frac{E_{\text {eqv }}}{E_{\text {crit }}}=1,
$$

where critical distention $\alpha_{\text {crii }}$ may be determined by the photomicrograph of spalled specimen; $E_{\text {crit }}$, however, may be obtained from measurements of tensile tests of notched tensile bars.

\section{SIMULATION OF TWO-DIMENSIONAL SPALLATION}

\subsection{Two-dimensional spall tests}

As an application of the foregoing theory, the processes of two-dimensional spallation in LY12 aluminum alloy are simulated. Three tests are performed with a $101 \mathrm{~mm}$ bore single-stage light gas gun [23]. The schematic arrangement for tests is shown in Fig. 5. Flyers and targets are machined into tapered and circular plates, respectively. All the experimental parameters are listed in Table 2. Pressure gauges are embedded in the target/PMMA (polymethylmethacrylate) interface on the symmetric axis to measure response for spall fracture in LY 12 aluminum alloy. In test 3, there is no pressure gauge embedded. The specimen of test 3 is soft recovered with a specially designed catcher to prevent any secondary damage. The recovered specimen is sectioned along the diameter into two parts for examination of the spall surface (shown in Fig. 8).

\subsection{Pressure-volume and stress-volume relations}

The stress-strain relations for material undergoing damage account for the presence of voids. As usual, the stress is separated into pressure and deviatoric components.

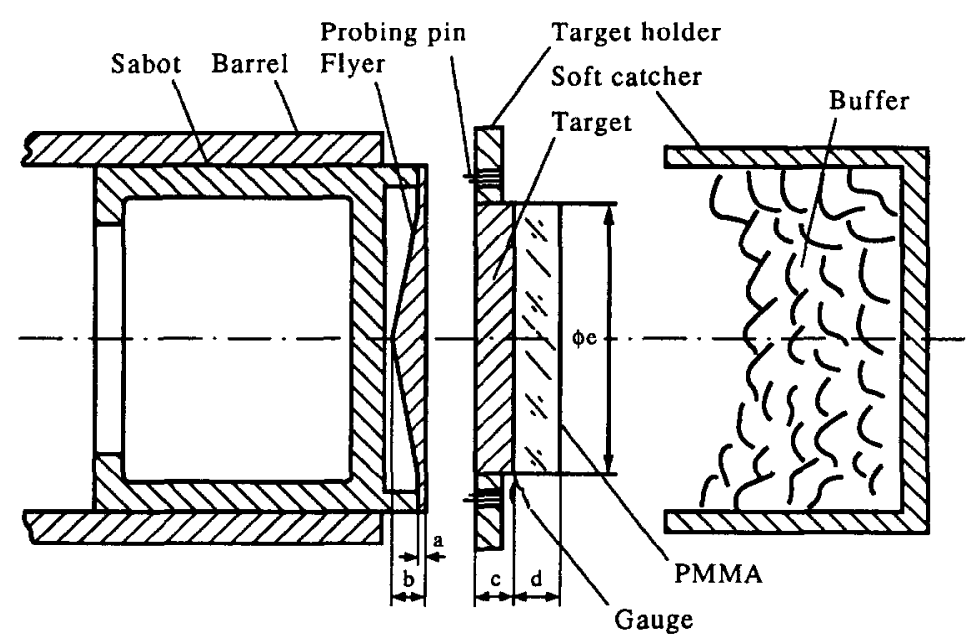

Fig. 5. Plate impact apparatus for two-dimensional spall study. 
The pressure is related to the specific volume and internal energy through a combination of the Mie-Gruneisen equation of state for the solid and a relation between pressure in the solid and average pressure on the porous material as assumed in previous section, i.e. eq. (1). In our numerical calculations, a small-strain form of the Mie-Gruneisen equation is used,

$$
P_{\mathrm{S}}=K_{0}\left(\frac{\alpha}{V}-1\right)+\Gamma \rho e_{\mathrm{S}}
$$

wherc $K_{0}$ is the bulk modulus, $\Gamma$ is the Gruneisen ratio and $\rho$ is the solid density.

The deviatoric stresses are computed by the usual elastic and plastic relations. However, the damage that occurs is presumed to affect both the yield strength and the effective shear modulus of the material. Relations between the effective shear modulus $\mu$, the yield strength $Y$ and distention $\alpha$ are given by [6]

$$
\begin{gathered}
\mu=\frac{\mu_{\mathrm{S}}}{\alpha}\left(1-\frac{6 K_{0}+12 \mu_{\mathrm{S}}}{9 K_{0}+8 \mu_{\mathrm{s}}} \frac{\alpha-1}{\alpha}\right) \\
Y=Y_{0} / \alpha .
\end{gathered}
$$

where $\mu_{\mathrm{S}}$ and $Y_{0}$ are the shear modulus and the yield strength of undistended material.
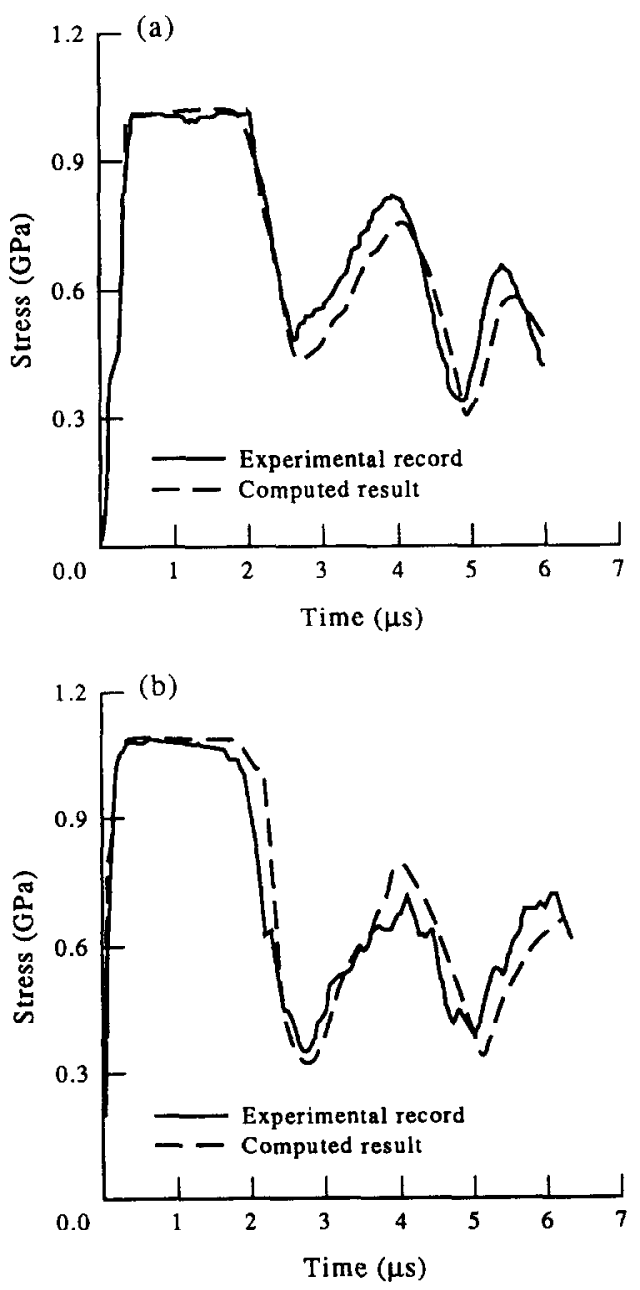

Fig. 6. Comparison of stress record from pressure gauges in PMMA behind LY12 aluminum alloy with computed stress. (a) Test 1 , (b) test 2. 


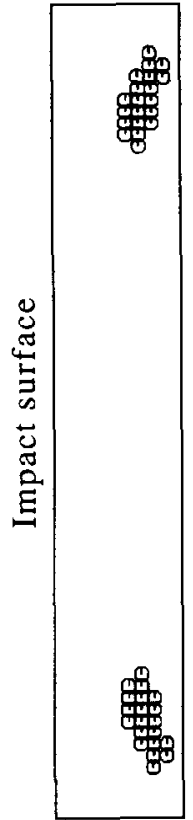

Time $=2.50(\mu \mathrm{s})$

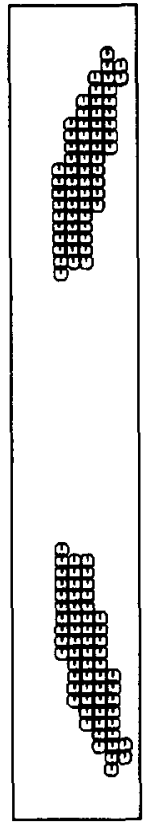

Time $=2.91(\mu \mathrm{s})$

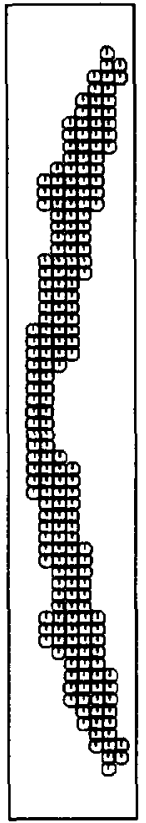

Time $=3.06(\mu \mathrm{s})$

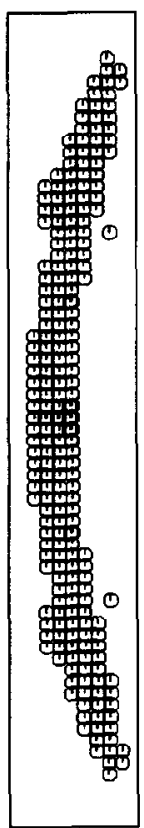

Time $=3.11(\mu \mathrm{s})$

Fig. 7. Sequence of cross-sections of LY 12 aluminum specimen showing progress of damage (not fracture) resulting from a tapered flyer impact: test 3 .

\subsection{Comparison of calculations with data}

Comparison of change of computed stress in PMMA with experimental record is shown in Fig. 6. Application of the dynamic fracture model to the problem of time-dependent spallation in LY12 aluminum alloy gives very good representation of the data.

In our fracture process, there are no large cracks, only microvoids. However, large cracks may be formed by the coalescence of small voids. In such cases, our method may be able to simulate the appearance and propagation of damage and large cracks. The damage actually occurs as a spall plane, which proceeds across the plate. The computed progress of this damage is shown in Fig. 7. The final computed position of the spall plane in the target is shown in Fig. 8(a), which is in agreement with the experimental data.

\section{SUMMARY}

A model of dynamic ductile fracture under intense dynamic general loading is developed which works well in describing two-dimensional spallation of LY12 aluminum alloy. One of the major differences in the model proposed here, as compared to other microscopic descriptions of dynamic ductile damage $[3,5,6,11]$, is that not only the external tensile pressure $P$, but also the external deviatoric stress $\Sigma_{\text {eqv }}$ (the effective stress) is taken into account for the void growth and compaction. A threshold stress (the combined action of hydrostatic and deviatoric stress) as a function of the current distention is directly obtained. Another feature of the model is that the influence of the inertial effects on the void growth is included. Numerical analysis suggests that the inertial effects appear to resist the growth of voids. It is significant at high strain rates and cannot be neglected.

Experimental observations $[12,13,20]$ show that flow localization is a very important factor leading to failure initiation in solids. In the present work, a simple criterion for ductile fracture is adopted which includes the contribution of both distention and plastic deformation. Although this criterion of fracture is oversimplified, it considers the evidence that plastic deformation plays a very important role in the process of dynamic fracture in most ductile materials.

The theoretical model proposed in the present paper is incorporated in a hydrodynamic two-dimensional finite-difference computer code, to simulate the processes of two-dimensional 
spallation in LY12 aluminum alloy. Comparison of numerical calculations with experimental results shows that the model describes the behavior of dynamic damage in specimens successfully.

\section{REFERENCES}

[1] T. W. Barber, L. Seaman, R. C. Crewdson and D. R. Curran, J. Mater. 7, 393-401 (1972).

[2] D. R. Curran, D. A. Shockey and L. Seaman, J. appl. Phys. 44, 4025-4038 (1973).

[3] L. Seaman, D. R. Curran and D. A. Shockey, J. appl. Phys, 47, 4814-4826 (1976).

[4] D. R. Curran, L. Seaman and D. A. Shockey, Phys. Today 30, 46-55 (1977).

[5] M. M. Carroll and A. C. Holt, J. appl. Phys. 43, 1626-1636 (1972).

[6] J. N. Johnson, J. appl. Phys. 52, 2812-2825 (1981).

[7] S. Cochran and D. Banner, J. appl. Phys. 48, 2729 (1977).

[8] A. M. Rajendran, M. A. Dietenberger and D. J. Grove, J. appl. Phys. 65, 1521-1527 (1989).

[9] M. A. Nash, RARDE Report 2/85 (1985).

[10] M. A. Nash and I. G. Cullis, Proc. 3rd Conf. Mech. Props High Rates of Sirain (pp. 307-314), Oxford (1984).

[11] R. Cortes, Int. J. Solids Structures 29, 1339-1349 (1992).

[12] D. R. Curran, L. Seaman and D. A. Shockey, Phys. Rep. 147, 254-388 (1987).

[13] M. A. Meyers and C. T. Aimone, Prog. Mater. Sci. 28, 1-96 (1983).

[14] D. E. Grady, J. Mech. Phys. Solids 36, 353-384 (1988).

[15] J. R. Rice and D. M. Tracey, J. Mech. Phys. Solids 17, 201-217 (1969).

[16] A. L. Gurson, J. Engng. Mater. Technol. 99, 2-15 (1977).

[17] A. C. F. Cocks, J. Mech. Phys. Solids 37, 693-715 (1989).

[18] J. M. Duva and J. W. Hutchinson, Mech. Mater. 3, 41-54 (1984).

[19] W. Johnson and P. B. Mellor, Engineering Plasticity. Van Nostrand Reinhold, London (1973).

[20] J. W. Hancock and A. C. Mackenzie, J. Mech. Phys. Solids 24, 147-169 (1976).

[21] D. A. Shockey, L. Seaman, K. C. Dao and D. R. Curran, J. Pressure Vessel Technol. 102, 14-21 (1980).

[22] J. N. Johnson and F. L. Addessio, J. appl. Phys. 64, 6699-6712 (1988).

[23] S. D. Zhao, L. T. Shen and S. L. Zhao, Acta Armamentaii 4, 49 (1985).

(Received 26 June 1993) 